\title{
Congenital Penil Lymphedema in a Preterm Neonate
}

\author{
(1) Şahin Hamilçıkan, @ Emrah Can \\ University of Health Sciences, Bağcllar Training and Research Hospital, Clinic of Pediatrics, Istanbul, Turkey
}

\begin{abstract}
Congenital penile lymph edema is a rare anomaly of unknown cause of the lymphatic vessels. Lymphedema is often the shortage of lymphatic collecting ducts, lymphatic hypoplasia, hyperplasia, as a result of aphasia or lymph node fibrosis associated with the pathological development of lymphatic vessels. Lymphedema is generally a permanent condition and surgical treatment is necessary. Here we present the case of a newborn at 26 weeks via caesarean section with congenital penile edema, and no detection of chromosomal anomalies.

Keywords: Preterm, lymphoedema, genital edema
\end{abstract}

\section{Introduction}

Congenital lymphedema is a rare pathology characterized by the abnormal development of the lymph vessels. Although the incidence below age 20 is predicted to be 1.15/100.000, frequency in newborns, especially in preterm neonates is unknown. Lymphedema consists of various lymph vessel pathologies including hypoplasia, hyperplasia, aplasia and lymph node fibrosis, and can be divided into two main groups as acute or chronic lymphedema $(1,2)$. In this article, we present a preterm neonate with congenital penile lymphedema which became notable 3 days after birth and lessened in the following weeks.

\section{Case Report}

A neonate weighing 960 grams was delivered by Caesarean section in the $26^{\text {th }}$ gestation week. Patient history revealed no antenatal follow-up. Appearance, Pulse, Grimace, Activity, and Respiration scores were 5 and 8 in the first and fifth minutes of birth respectively. He was admitted to neonatal intensive care unit (NICU) for Respiratory Distress syndrome and started on ampicillin and gentamicin treatment.
On the third day of hospitalization, significant penile edema was detected. He had no history of urinary catheterization prior to edema; and urine analysis and protein levels obtained by bag urine collection, urinary system ultrasonography, blood cell count and blood biochemical analysis showed no pathology. On the third day the patient developed a fever of 37.8 degrees but continued to receive the same antibiotic regimen. Acute phase reactants were negative. Despite the fact that initially penile edema was thought to be related to clinical sepsis, persistence in the following days led us to consider differential diagnosis, and the patient was referred to the urology department for urethra related pathologies, to pediatric nephrology for other urinary tract abnormalities, to pediatric surgery and pediatric endocrinology for Turner syndrome, hypothyroidism and related disorders. Cow's milk specific immunoglobulin E (lgE) and egg yolk specific lgE were evaluated after the pediatric allergy consultation and found within normal limits. Provocation test was used to further differentiate cow's milk allergy but no clinical response was observed. Penile venous Doppler ultrasonography showed no pathology but the patient continued to have penoscrotal swelling in the following weeks. Primary lymphedema was considered as a diagnosis of exclusion. When the patient was discharged from NICU at the $33^{\text {rd }}$ week of gestation, he 
weighed 2000 grams and had no other complaints (Figure 1). During the outpatient follow-up, he was consulted at the genetics department and chromosome analysis revealed no pathology. Tandem mass spectrometry, blood amino acid, and urine organic acid showed no sign of any metabolic diseases. Toxoplasma, others, rubella, cytomegalovirus, herpes screening resulted negative. Cranial ultrasonography and optometry were found normal. Penile edema started to resolve after 4 months and the patient was fully recovered after 6 months of follow-up. Written informed consent from a parent was obtained for this neonate prior to the study.

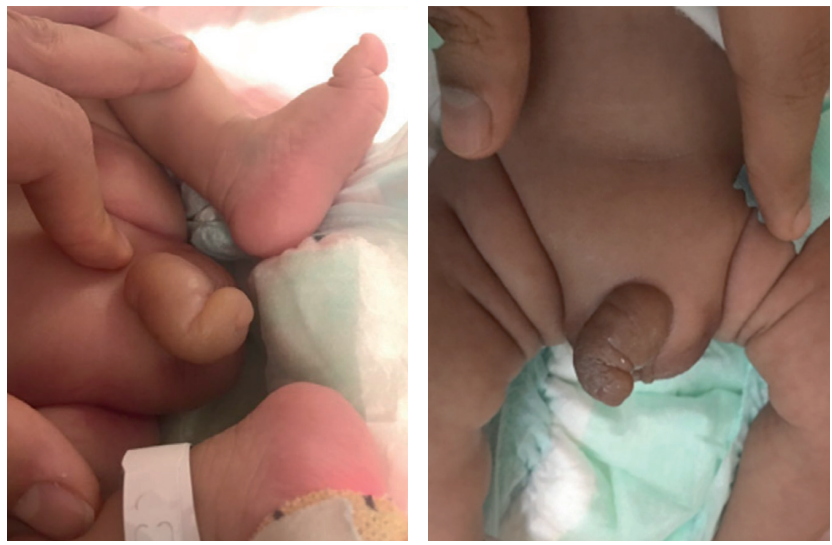

Figure 1. Congenital penil lymphedema in a neonate

\section{Discussion}

Congenital lymphedema is an autosomal dominant disorder and constitutes $10-20 \%$ of the lymphedemas $(3,4)$. Our case had no history of similar cases, and there was no consanguineous marriage in the family. Congenital lymphedema presents as edema of both extremities after birth and does not usually get worse. Milroy disease is also a cause of congenital lymphedema and it is seen in patients with family history. Patients frequently have edema of the lower extremities that sometimes reach out to the genitalia (5). Our baby showed no sign of lower extremity edema in the early postnatal period. He just had edema in the penile region, starting in the dorsum of the penis and continuing to the glans. He did not show any sign of lower extremity edema in the following days either. The diagnosis of congenital lymphedema is made by family history, especially urinary tract infections and similar cases in the family; and physical examination. There are no specific laboratory tests. The most significant point is to differentiate it from secondary lymphedema, therefore, other system evaluations, abdominal ultrasonography, urinary system evaluation and Doppler ultrasonography of iliac and inferior vena cava should be considered. For cases with edema of the lower extremities, genetic consultation is suggested. In our case, all these workups were performed and they revealed no positive results. Lymphangiogram is suggested for some complicated cases to make a definite diagnosis but application in newborns, especially in preterm neonates, is difficult $(6,7)$. We did not perform lymphangiogram in our case due to technical difficulties and the low success rate in children. Although lymphoscintigraphy has high sensitivity and specificity, we could not perform the test on our patient (5) as he was premature. Differential diagnosis of congenital penile edema are congenital lymphedema related Turner syndrome, Noonan syndrome, trisomy 13, 18 and 21. In our case consultation with the genetics department, and chromosomal analysis showed no pathology. Also, hamartomas in familial benign tuberosclerosis may present with lymphedema (8). Cranial and abdominal ultrasonography were performed on our case and no sign of hamartomas was found. The pathogenesis of primary congenital lymphedema due to congenital hypoplasia, deficiency or absence of superficial lymph system is not fully understood (8). One study revealed that the lymphatic system continued to develop after birth (4). From this point of view, it may be stated that idiopathic penoscrotal edema and congenital penile lymphedema are the same entities. Our case also promotes this finding as symptoms began to regress in the $4^{\text {th }}$ month and deteriorated in the $6^{\text {th }}$. No specific treatment is suggested for the cure of congenital lymphedema. The first and foremost step should be to prevent the progression of the lymph flow by conservative approaches. Appropriate treatment options are compression therapy and elevation. In severe cases abiding for a long period of time or with extra growth or dysfunction, surgery is the treatment of choice (5) although it has been suggested to evaluate each case for operation time and surgery technique $(4,5)$. In our case, surgery was not the first choice of treatment due to the prematurity of the baby, and the good response to conservative therapy. In conclusion, differential diagnosis of congenital lymphedema in newborns with penile edema should be kept in mind and careful evaluation should be done, especially in premature neonates.

\section{Ethics}

Informed Consent: Written informed consent from a parent was obtained for this neonate prior to the study.

Peer-review: Externally peer-reviewed.

\section{Authorship Contributions}

Surgical and Medical Practices: E.C., S.H., Concept: E.C., Design: E.C., Data Collection or Processing: E.C., S..H., Analysis or Interpretation: E.C., Ş.H., Literature Search: S..H., Writing: E.C., S..H.

Conflict of Interest: No conflict of interest was declared by the authors.

Financial Disclosure: The authors declared that this study received no financial support.

\section{References}

1. Bolt RJ, Peelen W, Nikkels PG, de Jong TP. Congenital lymphoedema of the genitalia. Eur J Pediatr 1998;157:9436. 
2. Smeltzer DM, Stickler GB, Schirger A. Primary lymphoedema in children and adolescents: a follow-up study and review. Pediatrics 1985;76:206-18.

3. Dale RF. The inheritance of primary lymphoedema. J Med Gen 1985;22:274-8.

4. Namir SA, Trattener A. Transient idiopathic primary penoscrotal edema. Indian J Dermatol 2013;58:408.

5. Schook CC, Kulungowski AM, Greene AK, Fishman SJ. Male genital lympedema:clinical features and management in 25 pediatric patients. J Pediatr Surg 2014;49:1647-51.
6. Greenlee R, Hoyme H, Witte M, Crowe P, Witte C. Developmental disorders of the lymphatic system. Lymphology 1993;26:156-68.

7. Fonkalsrud EW. Congenital malformations of the lymphatic system. Semin Pediatr Surg 1994;3:62-9.

8. Hoshiai S, Oguma E, Sato Y, Konishi T, Minami M. Congenital focal lymphedema as a diagnostic clue to tuberous sclerosis complex: report of two cases diagnosed by ultrasound. Skeletal Radiol 2015;44:1165-8. 